\title{
Research on LEACH-Based Wireless Sensor Network Routing
}

\author{
Junke Lv1,a \\ ${ }^{1}$ Xingzhi College, Zhejiang Normal University, Jinhua, Zhejiang, China
}

\begin{abstract}
Routing technology is one of the main supporting technologies of wireless sensor networks. Only by using routing algorithm reasonably or finding better routing optimization algorithm, can the function of wireless sensor networks be maximized. Therefore, the research of routing technology for wireless sensor networks has important theoretical and practical significance. Based on the analysis of existing routing protocols in wireless sensor networks, this paper focuses on LEACH protocol.
\end{abstract}

\section{Introduction}

Wireless Sensor Networks (WSNs) have been widely applied to battlefield investigation, rehabilitation therapy and field environment monitoring ${ }^{[1]}$. Numerous sensing nodes are deployed in the above-mentioned application environment. Sensing nodes can sense the environment data in real time and then transmit the data to the data management center so as to realize environment monitoring. However, due to limited energy supplied by sensing nodes, the energy consumption of these nodes is the key factor affecting the data collection efficiency of WSNs and shortening the network lifework. Once the nodes run out of energy, they cannot sense the environment data, which forms loopholes in WSNs coverage and shortens its lifetime $e^{[2-4]}$.

Consequently, it's the research focus to enhance the energy utilization of WSNs. Currently, the inter-cluster

\section{Problem description}

The Low Energy Adaptive Clustering Hierarchy (LEACH) protocol generates cluster heads at random and takes into account the residual energy of node. Specifically, a node (supposed as node $i$ ) generates randomly a uster head in this round if $\gamma>\lambda(i)$. The threshold of node $i$, namely $\lambda(i)$, is defined as shown in Equation (1):

$$
\lambda(i)= \begin{cases}\frac{p}{1-p \times\left(r \bmod \frac{1}{p}\right)}, & i \in G \\ 0, & i \notin G\end{cases}
$$

Where: $p$ represents cluster head proportion, namely the larger the number of cluster heads in networks, the greater the possibility that the node becomes a cluster head. $r$ represents the number of current round while $G$ indicates the set of nodes failing to become cluster heads in the previous round $(r-1)$. According to Equation (1), the nodes that have become cluster heads in the previous round are not considered as candidates for cluster heads in this round, aiming to balance node energy.

Notwithstanding the LEACH protocol takes into account energy balancing, there are still some shortcomings as follows:

(1) The threshold doesn't consider the residual energy of node. If a node with a little residual energy is chosen as the cluster head, its energy will be used up quickly, which makes direct impact on network lifetime;

(2) Under the LEACH protocol, the proportion of cluster heads $\mathrm{p}$ is fixed, which doesn't consider network energy distribution information;

(3) The spacing between nodes is not considered when setting the threshold. If the spacing is too large, the number of hops for data transmission will be increased and thus the energy consumption will be increased.

Thus, based on the LEACH protocol, the paper revises its threshold and meanwhile considers the heterogeneous sensor networks, namely there is a difference in the initial energy of node.

\section{Network model}

The network model ${ }^{[8-9]}$ shown in the Figure 2 can be considered, namely the source node forwards the data packet to the destination node (sink) through the relay nodes. The relay nodes between the source node and the sink nodes are also called forwarding nodes.

In addition, since the paper considers heterogeneous networks with three-level energy, namely node energy is divided into three levels and node energy varies from level to level, these nodes of various energy levels are respectively called advanced nodes, intermediate nodes and normal nodes. Advanced nodes contain highest

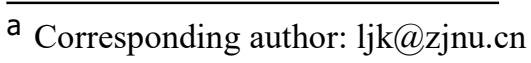


energy while normal nodes have lowest energy. These nodes of various levels have different proportion. It's supposed that the proportion of advanced nodes and intermediate nodes in network nodes are $m$ and $m_{0}$ respectively. It's supposed that the energy of normal nodes is $E_{0}$ and that of advanced nodes and intermediate nodes are represented by $E_{0}(1+\alpha), E_{0}(1+\beta)$ respectively, where $\alpha$ and $\beta$ are coefficients.

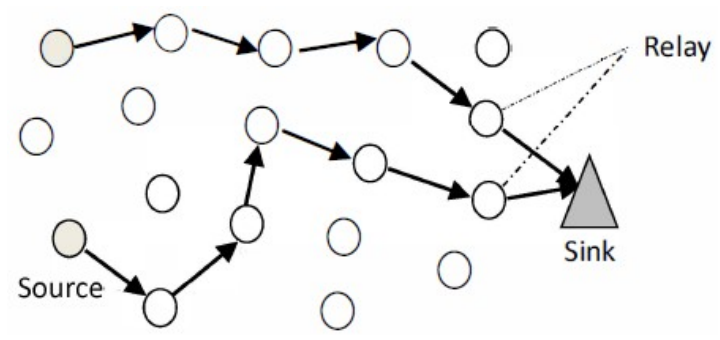

Figure 1. Network Model

\section{Performance simulation}

\subsection{Simulation parameters and performance indicator}

In order to better analyze the performance of the CTICR protocol, a simulation platform is established with the assistance of MATLAB and the LEACH and LEACH-D protocols are adopted as reference to compare their performance. It's supposed that 100 nodes are randomly distributed within $100 m \times 100 m$ network area. Specific simulation parameters are shown in Table 1. Each experiment is carried out independently for repeatedly 100 times and the average value is taken as the final experimental data.

Table 1. Simulation Parameter

\begin{tabular}{cc}
\hline Parameter & Value \\
\hline Simulation area & $100 \mathrm{~m} \times 100 \mathrm{~m}$ \\
Number of nodes & 80 \\
Node distribution & Random distribution \\
$E_{0}$ & $0.5 \mathrm{~J}$ \\
$E_{\text {elec }}$ & $50 \mathrm{~nJ} / \mathrm{bit}$ \\
$E_{\text {fris }}$ & $10 \mathrm{~nJ} /\left(\mathrm{bit} * \mathrm{~m}^{2}\right)$ \\
$E_{\text {tworay }}$ & $0.0013 \mathrm{pJ} / \mathrm{bit} / \mathrm{m}^{4}$ \\
$E_{D A}$ & $5 \mathrm{~nJ} / \mathrm{bit} / \mathrm{signal}$ \\
\hline
\end{tabular}

In addition, three indicators including network lifetime, stability duration and the number of data packet successfully received by the Base Station shall be adopted to assess the protocol. Network lifetime refers to the number of rounds when the last failure node occurs in the network; the stability duration refers to the number of rounds when the first failure node occurs in the network.

\subsection{Data analysis}

The CTICR protocol considers the heterogeneous networks with three-level energy. Thus, during the experiment simulation, $m=0.4, m_{0}=0.4$ while $\alpha=2$, $\beta=3.5$.

(1) Stability duration and network lifetime

The stability duration and network lifetime of the LEACH, DDEE and CTICR protocols are shown in Table 2. Data of 10 experiments are listed in Table 2.

Table 2. List of stability duration and network lifetime

\begin{tabular}{ccccccc}
\hline \multirow{2}{*}{ Experiment } & \multicolumn{3}{c}{ Stability duration (rounds) } & \multicolumn{3}{c}{ Network lifetime (rounds) } \\
\cline { 2 - 7 } & LEACH & DDEEC & CTTCR & LEACH & DDEEC & CTTCR \\
2 & 969 & 1355 & 1717 & 5536 & 5673 & 8638 \\
3 & 926 & 1355 & 1716 & 5553 & 5670 & 8637 \\
4 & 970 & 1342 & 1718 & 5537 & 5673 & 8636 \\
5 & 967 & 1357 & 1716 & 5532 & 5674 & 8643 \\
6 & 972 & 1365 & 1714 & 5538 & 5676 & 8640 \\
7 & 945 & 1358 & 1720 & 5539 & 5675 & 8640 \\
8 & 936 & 1350 & 1718 & 5530 & 5668 & 8638 \\
9 & 978 & 1355 & 1719 & 5534 & 5670 & 8640 \\
10 & 976 & 1359 & 1716 & 5538 & 5673 & 8641 \\
& 969 & 1355 & 1716 & 5530 & 5675 & 8641 \\
\hline
\end{tabular}

According to Table 2, the proposed CTICR protocol has the longest stability duration reaching 1,717 rounds while that of the LEACH and DDEEC protocols are 969 rounds and 1,355 rounds respectively. The cause is that the CTICR protocol reduces the energy consumption and balances network energy consumption through revising the threshold so as to prolong the stability duration. In addition, according to Table 2, the CTICR protocol has the longest network lifetime, far higher than that of the LEACH protocol.

It's not hard to figure out that the CTICR protocol can effectively improve network utilization rate, balance energy consumption and prolong network lifetime according to the experimental data. The mainly cause is that the CTICR protocol always attempts to choose the optimal node as the cluster head in each round so as to balance the energy consumption.

\section{Conclusion}

Data-centric wireless sensor networks have limited computing power, limited storage capacity, limited wireless communication capacity and limited power supply capacity. How to obtain as much and effective feature information as possible and transmit it to user nodes for processing in such a limited resource environment is the focus of current research. Therefore, designing energy-efficient routing strategies to prolong the lifetime of wireless sensor networks has become a key issue. Based on the analysis of existing routing protocols in wireless sensor networks, this paper focuses on LEACH protocol.

\section{References}

1. X.L. Xue. Research on Multi-hop Non-uniform Clustering Routing Algorithms for WSN Based on LEACH Protocol [D]. School of Information, East China University of Technology, (2010).

2. L.M. Sun, C. Ye, Y. Liao. Routing Mechanism for Sensor Networks [J]. Computer Science, 31(1) 54-57, (2004). 
3. X.Y. Mo. Wireless Sensors Research and Design of Network Clustering Routing Protocols [D]. School of Information, Zhejiang University, (2006).

4. X. Zheng, Research and Optimization of Energy Consumption Performance of LEACH-based
Wireless Sensor Networks Routing Protocols [D]. Shanghai: Department of Computer Science and Engineering, Shanghai Jiaotong University. 